\title{
Looking beyond the Sella: Suprasellar Infundibular Prolactinoma
}

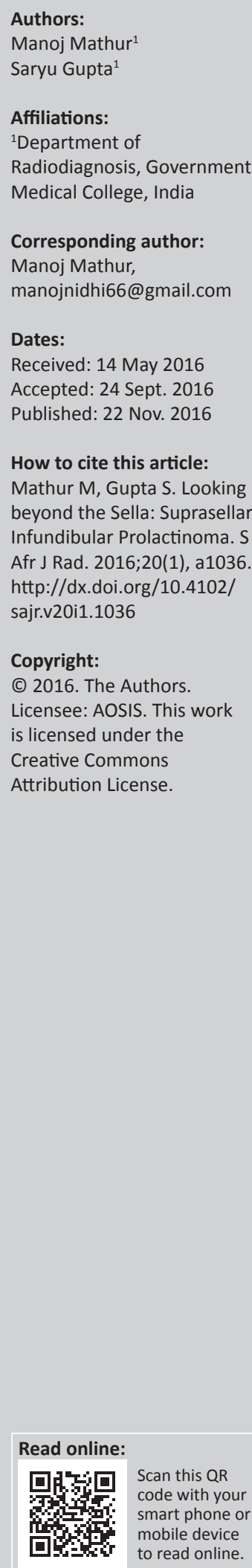

This is a case report of a 24-year-old female who presented with galactorrhoea and elevated serum prolactin levels of $150 \mathrm{ng} / \mathrm{mL}$. The intrasellar pituitary gland demonstrated normal morphology and characteristic enhancement. The pituitary stalk was thickened and revealed a non-enhancing lesion on dynamic contrast imaging, which was interpreted as a functioning microadenoma. The patient exhibited significant clinical improvement after initiation of therapy with a dopamine agonist, Cabergoline.

\section{Introduction}

Typically, pituitary adenomas are intrasellar in location, but infrequently, pituitary tumours may occur ectopically within pituitary rests. A pituitary tumour is considered ectopic when the adenoma has no communication with intrasellar pituitary gland. ${ }^{1}$ Ectopic pituitary adenoma was first described by Erdheim in 1909. ${ }^{2}$ These tumours may be functioning or non-functioning. Functioning tumours tend to be diagnosed earlier because of the hormonal imbalances they induce. The non-functioning tumours present with symptoms related to local mass effect on neighbouring structures and therefore present later.

The hypothalamus and pituitary gland act synergistically via the hypothalamic-pituitary axis. The hypothalamus stimulates the release of all other hormones of the anterior pituitary except for prolactin (PRL). The secretion of PRL is inhibited by the hypothalamus, and this negative regulatory effect on prolactin is mediated via the release of dopamine into the hypothalamichypophyseal portal vessels. Both intrasellar and ectopic prolactinomas are usually under feedback regulation by dopamine and its analogues.

The principal effects of PRL are its role in lactation and reproduction. This article documents a report of an ectopic functioning pituitary microadenoma (microprolactinoma), diagnosed at magnetic resonance imaging (MRI).

\section{Case report}

A 24-year-old female patient presented with headache, galactorrhoea and menstrual irregularity. Her PRL levels were elevated at $150 \mathrm{ng} / \mathrm{mL}$ (normal reference range is 3-24 ng/mL). Growth hormone and thyroid-stimulating hormone levels were normal. There were no visual field defects on perimetry measurements and the visual acuity was normal. Radiographs of the cranium were normal and a MRI was requested.

T1-weighted non-contrast MRI demonstrated thickening of the pituitary stalk (Figure 1). Dynamic contrast-enhanced T1 sagittal (Figure 2a) and coronal (Figure 2b) images of the sellar and parasellar regions revealed a hypoenhancing microadenoma in the anterior aspect of the pituitary stalk. This tumour was not connected to the intrasellar pituitary gland. The intrasellar pituitary gland was normal in height and signal intensity on both pre- and post-contrast images with a flat superior contour. The parasellar regions were normal bilaterally. The optic chiasm displayed normal morphology with no evidence of compression, and the sphenoid sinus and clivus were normal in appearance.

A diagnosis of an ectopic pituitary microadenoma within the pituitary stalk was made. In view of the elevated PRL levels, the adenoma was assumed to be a microprolactinoma and medical treatment with Cabergoline was initiated. The patient's PRL levels decreased to $20 \mathrm{ng} / \mathrm{mL}$ after 4 months of treatment. However, a follow up MRI performed at 4 months revealed no regression in the size of the microadenoma (Figure 3). As reduction in PRL levels often predates tumour regression, the patient continued treatment with a maintenance dose of Cabergoline. 


\section{Discussion}

Ectopic pituitary adenomas can occur in the suprasellar region, sphenoid sinus, cavernous sinus and clivus. ${ }^{3}$ The most common location for an ectopic adenoma is the sphenoid sinus, followed by the suprasellar region. ${ }^{3,4}$ The ectopic location of sphenoid sinus adenomas can be explained by the fact that the Rathke's pouch takes its origin from the primitive

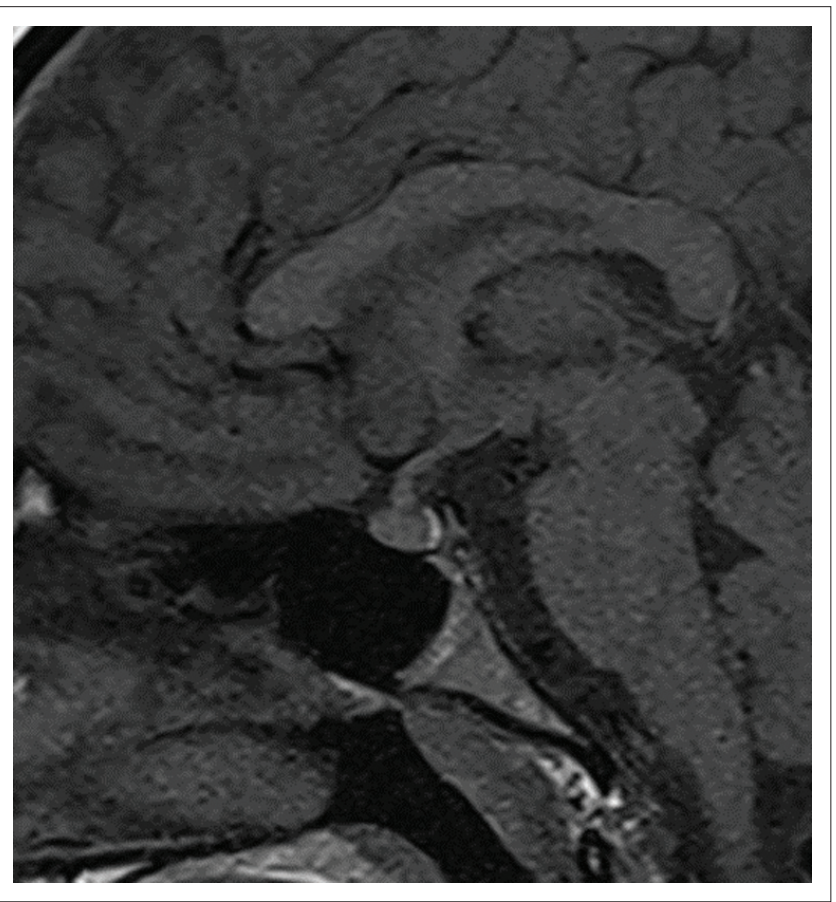

FIGURE 1: Unenhanced T1-weighted image reveals thickening of the pituitary stalk. The adeno and neurohypophysis are visualised in their normal intrasellar location. pharynx and ascends cranially to form the anterior pituitary. The posterior pituitary and median eminence of the hypothalamus are derived from the neuroectoderm at the base of the forebrain, and the infundibulum or pituitary stalk originates from the ventromedial hypothalamus. As the stalk enters the pituitary, it is enveloped by the pars tuberalis, which is a part of the anterior pituitary.

Stalk adenomas are postulated to arise from pituitary cell rests within the arachnoid layer covering the stalk. ${ }^{5}$ Alternatively, they can arise from pars tuberalis which covers the stalk anteriorly, close to the pituitary insertion. These cell rests have the potential to develop into an adenoma, even though they do retain the negative feedback regulation by dopamine and its analogues. ${ }^{6}$

On unenhanced scans, stalk adenomas manifest as thickening of the stalk. The normal stalk measures $3.25 \pm 0.56 \mathrm{~mm}$ at the level of optic chiasma and tapers down to measure $1.91 \pm 0.4$ $\mathrm{mm}$ at its pituitary insertion. ${ }^{7}$ After the administration of gadolinium, these tumours typically demonstrate hypo or delayed enhancement, relative to the normal pituitary, and dynamic contrast-enhanced MRI is the imaging modality of choice for their diagnosis.

Alternative aetiologies for stalk thickening include tumours (germinoma, lymphoma and leukaemia), granulomatous conditions (Langerhans cell histiocytosis, neurosarcoidosis and tuberculosis) and inflammatory conditions (infundibuloneurohypophysitis). ${ }^{7}$ Turcu et al. studied 152 cases of pituitary stalk lesions in a Mayo clinic review study and defined the role of clinical history, biochemical tests and MRI morphology in characterising these lesions. ${ }^{8}$

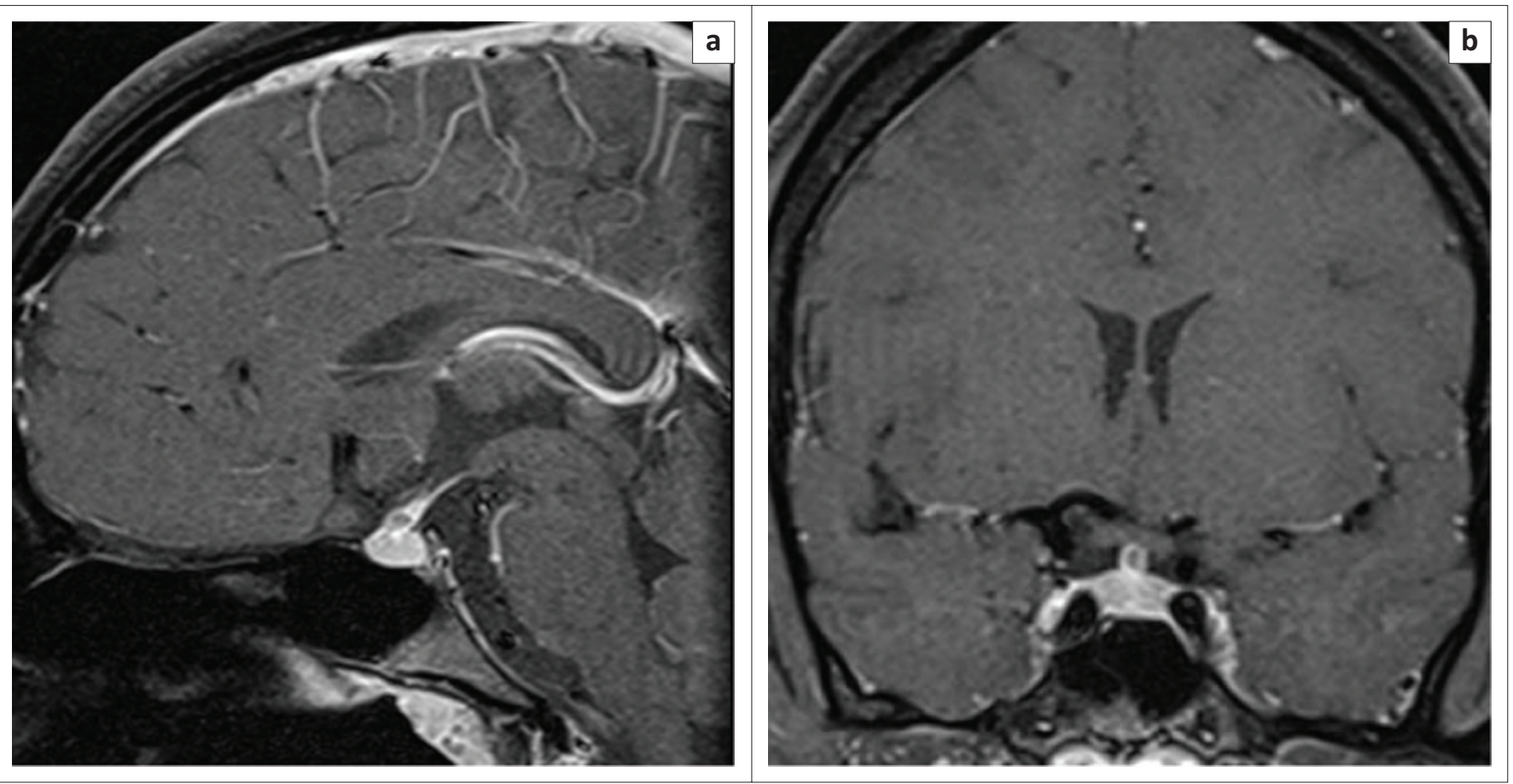

FIGURE 2: (a) Sagittal post-contrast T1-weighted image defines the adenoma in the distal part of the thickened pituitary stalk. The adenoma enhances less than the stalk and the gland; (b) Coronal dynamic contrast-enhanced image reveals a hypo enhancing mass in the pituitary stalk. There is no significant compression of the optic chiasm. 


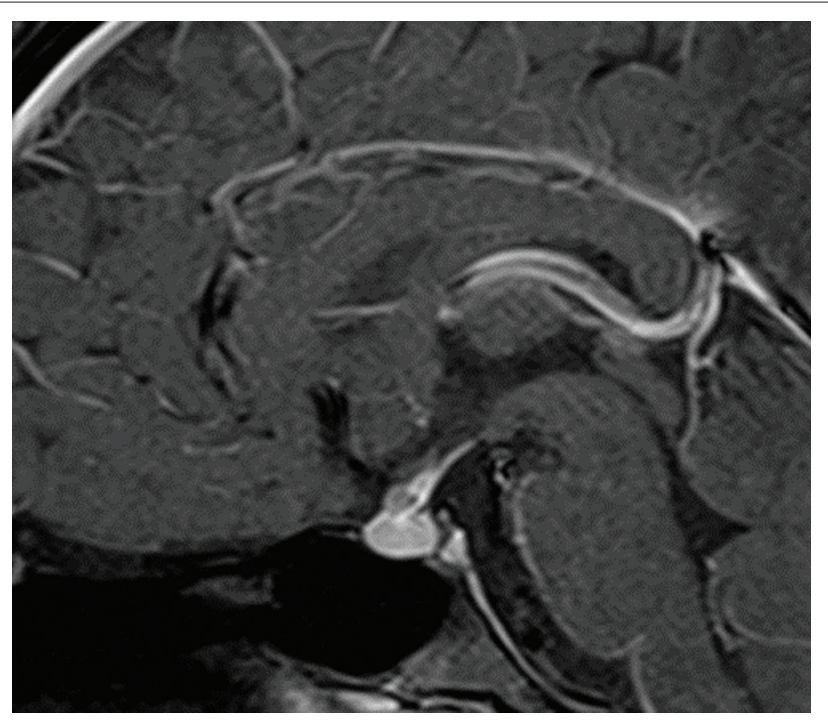

FIGURE 3: Contrast-enhanced MRI performed at an interval of 4 months after initiation of medical treatment with Cabergoline revealed no significant regression in the size of adenoma, despite the prolactin levels having returned to normal. The decrease in hormone levels usually precedes the reduction in tumour size.

As dopamine inhibits the release of PRL by the anterior pituitary, its analogues, Bromocriptine and Cabergoline form the mainstay in the medical management of prolactinomas. This treatment also reduces the size of the tumour. Following treatment, the PRL levels decrease in a matter of days to weeks and precede the tumour shrinkage. This was also observed in our case. Surgical treatment is reserved for patients who are refractory to Cabergoline or in the presence of significant pressure effect on the optic chiasm.

\section{Conclusion}

For a holistic evaluation of the pituitary gland, it is imperative that we examine beyond the sella so that adenomas lurking in ectopic locations do not remain undetected. Ectopic pituitary adenomas are best diagnosed by dynamic contrastenhanced MRI. Awareness of the various ectopic locations assists in making the correct diagnosis.

\section{Acknowledgements Competing interests}

The authors declare that they have no financial or personal relationships, which may have inappropriately influenced them in writing this article.

\section{Authors' contributions}

M.M. was the principal investigator who diagnosed the case and did subsequent follow up. S.G. assisted in the write up of the case and helped in follow up as well.

\section{References}

1. Matsuno A, Katayama $H$, Okazaki R, et al. Ectopic pituitary adenoma in the sphenoid sinus causing acromegaly associated with empty sella. ANZ J Surg. 2001;71:495-498. http://dx.doi.org/10.1046/j.1440-1622.2001.02173.x

2. Erdheim J. Über einen Hypophysentumor von ungewöhnlichem Sitz. Beitr Pathol Anat. 1909;46:233-240.

3. Appel JG, Bergsneider M, Vinters H, Salamon N, Wang MB, Heaney AP. Acromegaly due to an ectopic pituitary adenoma in the clivus: case report and review of literature. Pituitary. 2012;15 Suppl 1:S53-S56. http://dx.doi.org/10.1007/s11102-011-0345-9

4. Hong JF, Ding XH, Wang SS. Coexistence of ectopic pituitary adenoma and empty sella in a patient with acromegaly: A case report and review of literature. Neuro India. 2012;60:304-306. http://dx.doi.org/10.4103/0028-3886.98517

5. Ueda R, Katayama M, Yoshida K, Kawase T. Suprasellar peri-Infundibular ectopic prolactinoma. Neurol Med Chir (Tokyo). 2003;43:51-54. http://dx.doi.org/10. 2176/nmc.43.51

6. Yang BT, Chong VFH, Wang ZC, Xian JF, Chen QH. Sphenoid sinus ectopic pituitary adenomas: CT and MRL findings. BJR. 2010;83:218-224. http://dx.doi. org $/ 10.1259 / \mathrm{bjr} / 76663418$

7. Hamilton BE, Salzman KL, Osborne AG. Anatomic and pathologic spectrum of pituitary infundibular lesions. AJR. 2007;188:W223-W232. http://dx.doi.org/10. 2214/AJR.05.2027

8. Turcu AF, Erickson BJ, Lin E, et al. Pituitary stalk lesions: the Mayo Clinic experience. $\mathrm{J}$ Clin Endocrinol Metab. 2013;98(5):1812-1818. http://dx.doi.org/10.1210/jc. 2012-4171 